\title{
Metrology applications to D\&D issues: issues at stake for INSIDER European project
}

\author{
Danièle Roudil ${ }^{1, *}$, Marielle Crozet $^{1}$, Sébastien Picart $^{1}$, Ben Russell ${ }^{2}$, Margarita Herranz ${ }^{3}$, Sven Boden ${ }^{4}$, \\ Paolo Peerani ${ }^{5}$, and Laura Aldave De Las Heras ${ }^{5}$ \\ ${ }^{1}$ CEA, DEN, DMRC, University of Montpellier, Marcoule, France \\ ${ }^{2}$ Nuclear Metrology Group, National Physical Laboratory, Hampton Road, Teddington TW11 0LW, UK \\ 3 Nuclear Engineering Department, University of the Basque Country (UPV/EHU), Pza. Ingeniero. Torres Quevedo, 1, \\ 48013 Bilbao, Spain \\ 4 SCK-CEN, DDW, Boeretang 200, 2400 Mol, Belgium \\ ${ }^{5}$ European Commission, Joint Research Centre (JRC), Directorate G - Nuclear Safety \& Security, Rue du Champ de Mars 21 , \\ 1050 Brussels, Belgium
}

Received: 13 September 2019 / Accepted: 4 November 2019

\begin{abstract}
Nuclear metrology is an essential aspect to consider for further improvements of the initial characterization of sites under decommissioning. The H2020 Euratom project INSIDER in June 2017 aims at improving the management of contaminated materials arising from decommissioning and dismantling (D\&D) operations by proposing an integrated methodology for radiological characterization. This methodology is based on advanced statistical processing and modelling, coupled with adapted or innovative measurement methods. A metrological approach supports the qualification of this integrated methodology with a concrete application to real projects representative of the use cases identified in the project. Assessment of the outcomes will be used for providing recommendations and guidance resulting in pre-standardization texts.
\end{abstract}

\section{Introduction}

The Euratom treaty (1958) and the nuclear NonProliferation Treaty (NPT) (1970) specify the modalities of control and survey of nuclear materials.

Each step of the nuclear fuel cycle is concerned and has to follow regulatory or customer requirements.

In the field of nuclear energy production and associated fuel cycle, industrial recycling plants and R\&D facilities are subject to analytical monitoring to meet regulatory criteria and to ensure secure facilities, appropriate waste storage conditions and non-proliferating processes. Measurement results are only truly comparable when they include a well estimated uncertainty.

Control of nuclear materials is based on accurate knowledge of the quantities and grades of nuclear material present in the facilities and on material transfers between facilities. This knowledge is obtained by direct nondestructive measurements as well as by destructive sample analysis, which has the advantage of providing more accurate measurements of the material quantities. Safeguards verification is based on "operator-inspectors" comparisons.

\footnotetext{
* e-mail: danielle.roudil@cea.fr
}

New requirements are foreseen in relation to the likely evolution of the nuclear context, including on the one hand recycling of fuels with different compositions and on the other hand nuclear material analysis prior to decommissioning and dismantling (D\&D) [1]. In this context, European countries have to face and anticipate a big wave of reactor and nuclear facility shutdowns. The implementation of the decommissioning operations will have a huge impact on waste production, their cost and duration. An international consensus already exists on the importance of the characterization contributions from the front-end to the back-end of the global decommissioning process $[2,3]$.

A better understanding of measurement results and their interpretation is indispensable to progress in new approaches and methodologies. In this context, the contribution of metrology becomes essential.

One of the principal missions of the CEA's Committee for the establishment of analysis methods (CETAMA) is to promote best analytical practices in French and European nuclear laboratories, providing metrology support, in particular reference materials production and marketing, interlaboratory comparisons organization and uncertainty assessment [4].

CETAMA coordinates the European Euratom project INSIDER (Improved Nuclear Site Characterization for waste minimization in Decommissioning under 
Constrained environment) which is devoted to the development and qualification of integrated pre-characterization approaches, coupling sampling to on-site and in-lab measurements with respect to operational decommissioning efficiency, safety and costs. Increased confidence in the quantities and types of resulting waste is expected for subsequent storage and disposal end-points. This article illustrates the fundamental role that metrology can play in making this approach reliable and qualified.

\section{Metrological support in the nuclear field}

\subsection{Aims and purposes}

At each step of the fuel cycle (including fuel fabrication and spent fuel reprocessing, waste and effluent characterisation, material control and safeguards,) an analytical control is ensured by centralized industrial or R\&D laboratories. One of their missions is to guarantee the accuracy of analysis results for:

- quality control of products;

- control of material flow;

- nuclear material follow up and accountancy;

- safety and Environmental controls;

- U, Pu flow balance.

Analytical requirements are established at national levels and at European and international level by Euratom and the IAEA.

The analytical process stands for all the steps between the initial sampling up to the processing of the acquired data, including any chemical pre-treatment of the samples depending on the detection device chosen. Uncertainty is quantification of the level of confidence in the measurement result. Therefore the estimation of an uncertainty implies the knowledge of the complete measurement process and its influencing factors. Metrological tools, interlaboratory comparisons, certified reference materials (CRM), standards and methods, are essential to achieve this objective in a traceability approach perspective.

There is a wide range of uranium and plutonium isotopic certified reference materials provided in Europe mainly by JRC, NPL, PTB, CEA and radioactivity activity CRM (CEA, NPL). Structured traceability schemes is essential for the elementary and isotopic analysis of radionuclides (Fission and activation products, minor actinides, etc.) but difficult to set up as this requires that metrology laboratories have adequate facilities for the handling of nuclear materials. Some key institutes, such as the IAEA and national nuclear institutes have developed their own quality control tools.

The improvement of the certified reference materials values and uncertainties is one of the challenges of chemical metrology to face with future nuclear requirements. The controls need to be supported by accurate and comparable measurements which have to rely on reliable references. Indeed, long-term credible comparability can only be guaranteed by making all measurements traceable to the same long-term stable references. The development of nuclear CRM therefore has to be pursued and close cooperation between Reference Materials Producers must be strengthened.
The design of decommissioning and dismantling operations generally requires a large number of specific measurements and analyses. They must also allow the assessment of the generated waste. Onsite measurements are preferred for reasons of cost and speed. Laboratory analyses are particularly relevant for Difficult to Measure (DTM) radionuclides $(\mathrm{RN})$. Considering the wide variety of matrices and the large quantity of radionuclides potentially present in the samples, development and especially validation of improved measurement methods are very challenging. Facing the quantity of nuclear facilities to be shut down in the coming decades, this is a major technological, analytical and metrological issue.

\subsection{Metrological scheme}

\subsubsection{General considerations}

The BIPM (International Bureau of Weights and Measures) is the international reference organization for the world measurement system and aims to promote and move forward global comparability of measurements. It is supported by the International Committee for Weights and Measures (CIPM) and its Consultative Committees (CC).

The National Institutes of Metrology (NMI) and socalled designated laboratories (DI) are national bodies in charge of scientific and technical metrology in each country. They rely locally on Regional Metrology Organisations (RMOs). Their active members are the NMIs and DIs of the countries that have joined them.

As an example, the RMO for the Europe region, EURAMET (European Association of National Metrology Institutes), currently comprises 37 countries.

In the frame of nuclear chemical and radiochemical analysis, the principal missions of CETAMA, transverse unit of CEA, since 1961 is to promote best analytical practices in French and European nuclear laboratories, with a view to validating, to evaluating and/or to improving performances. It is also in charge of analytical innovation support.

The general functioning of the metrology developments and the CETAMA positioning is shown in Figure 1.

Based on a network of laboratories and experts structured in working groups and on its own metrology laboratory LAMMAN, the CETAMA provides reference materials to meet the analytical needs and organizes interlaboratory comparisons (ILC).

Realistic assessment of uncertainties results remains at the heart of analytical issues.

While the metrological scheme for laboratory analyses is well established, based on Certified Reference Materials (CRM) and ILC, additional work remains to be done for onsite measurements in the case of D\&D issues.

These works are generally finalized by a common synthesis of good practices and recommendations used as reference documents.

\subsubsection{Interlaboratory comparisons}

Different types of interlaboratory comparisons exist depending on the analytical objectives: 


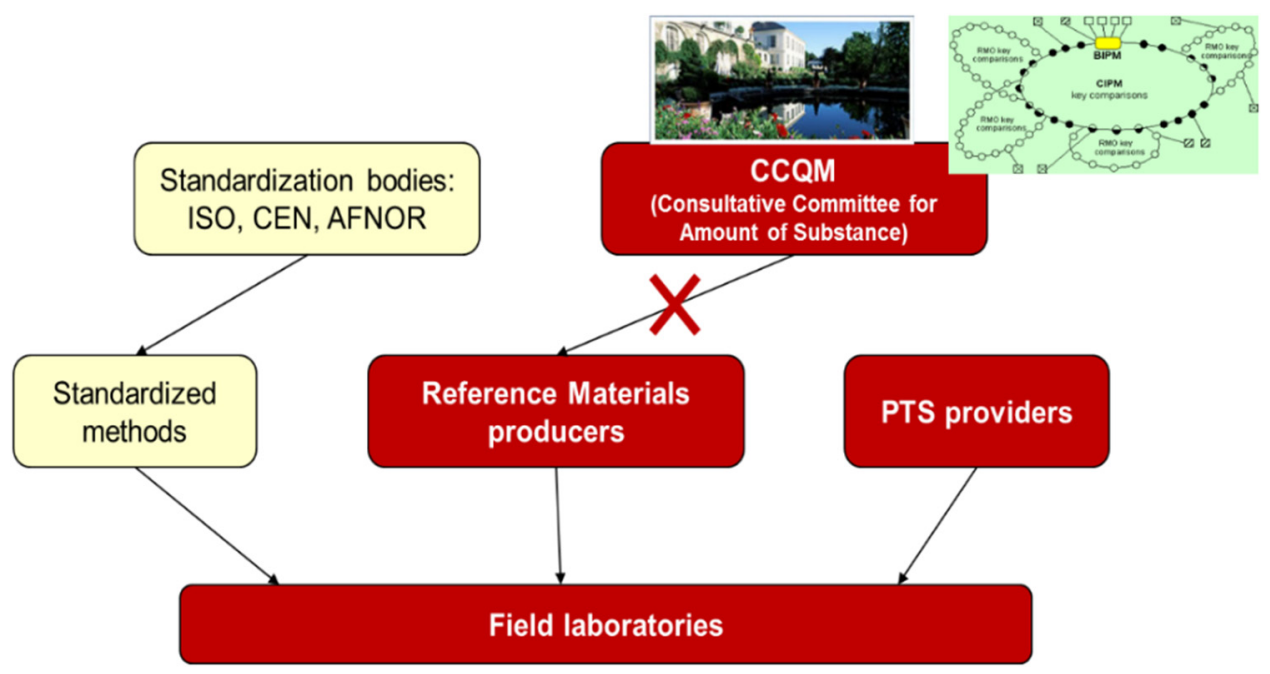

Fig. 1. Positioning of the functions of CRM producers and PT organisers in chemical and radiochemical metrology - CETAMA involvement. From [5].

- proficiency testing (PT) to evaluate and compare the laboratory performance scores;

- method validation comparison to evaluate the performance of a specific method;

- certification to assess the reference value(s) and associated uncertainty of a reference material.

Different techniques are widely used by the nuclear laboratories, applying validated methods. To improve the confidence in the analytical results, in particular their methods trueness, PTs offer periodic monitoring and evaluation of routine laboratory performance. European bodies, such as JRC Geel, IAEA Nuclear Material Laboratory (NML) for example, and at CEA, CETAMA and LNHB organized periodically different kind of PT, providing also laboratories with an opportunity to check for possible deviations and if necessary implement the necessary corrective actions.

The main advantages of PT interlaboratory comparisons are:

- the wide variety of matrices and analytes possibly covered by these tests;

- the possibility of method performance comparison owing to the fact of their number of exercises, and/or numerous participating laboratories and compliance to standards in force [6].

Availability of certified reference materials remains complementary, because of some disadvantages of PT compared to CRM:

- no mandatory warranties in terms of long-term stability

(outside the testing period) and traceability of the reference samples;

- they shouldn't be used for calibration.

\subsubsection{Certified reference materials}

Analysis laboratories rely on CRM for quality control of measurements, for calibration of analytical instrumentation, and for spiking of samples for elemental assay by isotope dilution analysis, to evaluate and validate the performance of their testing procedures:

- CRM (similar liquid, solid or gaseous matrix and liquid isotopic tracers) so that laboratories can characterize their method in real case;

- reference materials (RM) for precision;

- CRM for trueness.

In the field of nuclear fuel cycle, these CRM materials are used for the monitoring of nuclear materials, process controls, monitoring of the environment.

High chemical purity CRMs, reference solutions and sources are more particularly suitable for calibrating equipment and quality control monitoring.

Matrix CRMs are necessary to evaluate the performance of methods in terms of trueness and precision. However, to obtain traceable measurement results with very low uncertainties, isotopic standards or spikes are indispensable. Such materials are currently commercially available for uranium and plutonium.

In Europe the main producers in the nuclear sector are JRC Geel, IAEA, PTB, NPL, LEA and CEA. NIST and DOE/NBL CRMs are also widely used in the laboratories.

The lack of suitable available CRM for D\&D applications has been identified. Some specific developments were supported through the European Metrology Programme for Innovation and Research (EMPIR) projects such as Metrodecom I and II in particular, for an operational use of detectors.

CETAMA has offered different types of certified reference materials for more than 30 years, (High purity materials, matrix materials, isotopic composition).

The associated uncertainties of CRM certified value found in certificate are of main importance regarding calibration process and finally trueness of analysis results $[7,8]$.

Different statistical approaches are used to assess a reference value: arithmetic mean, weighted mean, robust mean and more recently the "Weighted mean and Excess variance" approach recommended by the international 

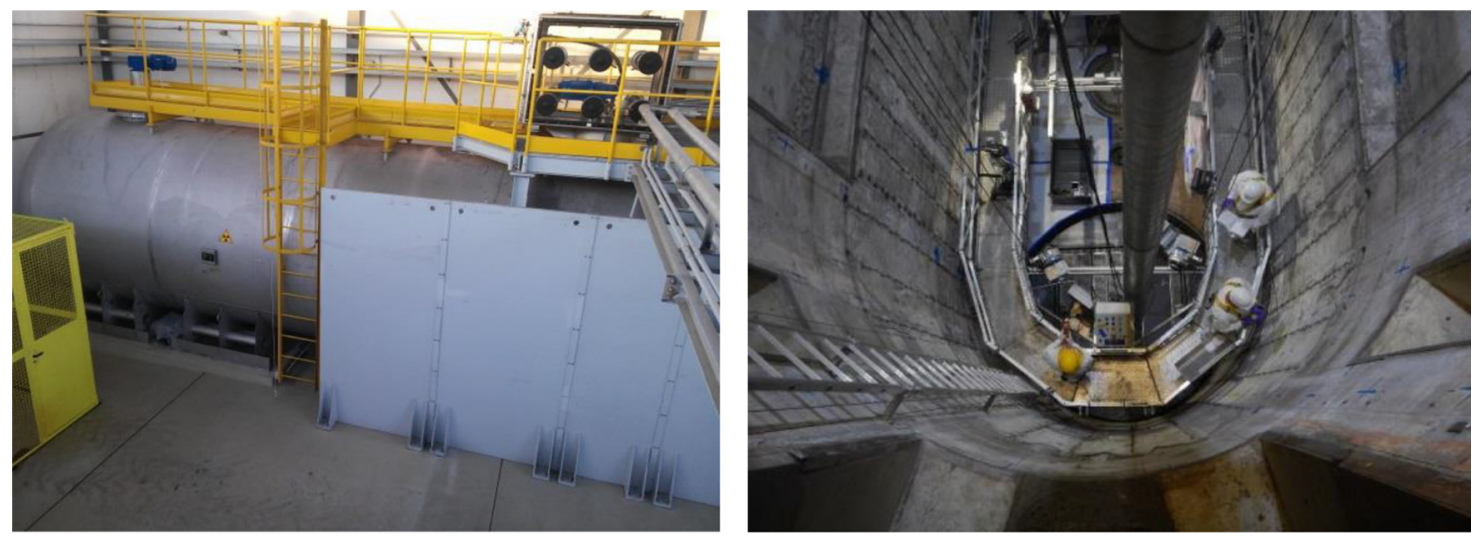

Fig. 2. Use case 1 and use case 2 INSIDER worksite: Effuent tank from JRC ISPRA, and Biological shield of BR3 Mol reactor from SCK-CEN.

metrology committee CCQM (Consultative committee for amount of substance). Uncertainties are determined accordingly $[9]$.

\subsubsection{Standards and guidelines}

Industrial projects need validated analytical methods and reliable measurement results, according to the standards in force: a validated method is a standardized one or a well characterized method in terms of trueness, precision, limit of detection/quantification, based on CRM use and on ILC participation.

Analytical method and reference methodological guide establishments are main achievements of metrological support of the CETAMA network, its WGs and sub-WGs. The standardization of the laboratory good practices is a main advantage considering time and cost of implementation. In this context different kind of $\mathrm{RN}$ analytical methods can be commonly validated (i.e. CETAMA WG) and proposed in support to standardization commissions (ISO, French BNEN).

Concerning methodological guides, the sampling and characterization ISO standard [10] devoted to the dismantling of nuclear site is based on a methodological report [11] applied to international decommissioning project management, combining sampling and measure after historical analysis. The power of geostatistical approaches for initial and intermediate characterization is highlighted through different examples and feedbacks [12]. This standard describes a common approach of both R\&D and industrial laboratories, and project leader.

Methodological guides were also produced recently by the WGs dedicated to analytical techniques, namely ICP, nuclear measurements, and radiochemistry [13-15].

Follow-up of liquid and gaseous releases into the environment or nuclear waste management may lead to the problematic evaluation of detection limits [16] as a sum of individual contributions. The guides of performance assessment for cumulative results should enable the laboratories to adopt a new common and reliable methodology for the calculation of cumulative measurement values, ensuring comparability and accuracy of the results they provide to their customers.

\subsection{Metrology perspectives}

In the framework of $\mathrm{D} \& \mathrm{D}$ projects, analysts are facing new technical challenges in terms of the number and size of samples, new and numerous kind of matrices and improvement of uncertainty evaluation.

The tools previously described and optimized in the framework of nuclear fuel cycle analysis should be used and adapted to these new problematics but also to regulatory and financial new requirements.

Analytical techniques and method performances are globally well studied and efficient tools exist for their evaluation. In-lab DTM radionuclide analysis methods would necessitate adaptation and improvements taking into account matrix effects and the potential interferents.

Most of these matrices are solid materials, which increase the difficulties of RM certification due to the homogeneity constraints to be respected.

Characterization of D\&D worksites is mostly based on different type of on-site measurement techniques. Equipment calibration and method performances in representative environment is not easily controlled.

International organizations had also highlighted a lack of guidelines and standards as well as feedback on past decommissioning projects.

Last but not least, future challenges for D\&D should lead in sampling strategy and associated data treatment to aim for efficiency at the right need and at the right cost. The reliability of the data that will feed these models becomes essential.

\section{Application to the INSIDER project}

\subsection{D\&D context and general methodology}

The design of D\&D operations is highly dependent on the history of nuclear installations and the radionuclide inventory implemented. These D\&D projects are potentially significant sources of radioactive waste (volume, type, category, etc.) and their management remains a technical, economic, financial and societal challenge and therefore a major focus for optimization. In an environ- 
ment constrained by the level of radiological activity or by the size of a site, for example, the development and validation of new sampling methodologies are necessary to access the estimation of the initial composition and volumes of contaminated materials in a shorter time and at a lower cost. This data will have a direct impact on the production of final waste and on the associated project planning.

The resulting mapping should allow a simulation of the radiological state of the facility (or components), corresponding to the predefined analytical objectives, in particular the level of accuracy required to support the choice of options for scenarios. A realistic evaluation of the performance of destructive and non-destructive methods of interest is therefore essential.

The European project called INSIDER has been accepted by the European Commission and launched the 1st of June 2017. INSIDER is coordinated by CETAMA (CEA Nuclear Energy Division) and includes 18 European partners from 10 member states [17]. In the frame of metrology, three European NMI $(N P L, C M I, L N E)$ and one DI ( $L N H B$ at $C E A$ ) are partners of the project. An End User Group (EUG) will provide user requirements and feedback from the beginning of the project.

The project focuses on radiological characterization, including the sampling strategy and sampling design, through waste-led integrated approaches. Its objectives are thus to improve the management of waste coming from nuclear sites or facilities under D\&D with medium (MA) and high radioactivity (HA) levels and/or other constrained environments with respect to operational decommissioning efficiency, safety and costs. It will increase confidence in the quantities and characteristics of resulting radioactive waste in particular concerning subsequent storage and disposal end points, and could lead to more reliable risk analyses.

The INSIDER strategy promotes an integrated and overall approach of pre-decommissioning characterization, which notably consists in evaluating historical data, making on-site measurement campaigns, sampling and analysing, developing scaling factors and applying numerical codes. Therefore, a strategy for sampling in the field of initial nuclear site characterization in view of decommissioning, is offered to guide the end user to appropriate statistical methods and approaches to use for data analysis and sampling design [18]. A selection of available fit for purpose measurement methods will be reviewed in the frame of experimental benchmarks.

Many analytical innovative developments, particularly for in-situ measurements, have been supported by national and European projects in recent years in order to give access more rapidly to measurement values or to provide specific imaging tools adapted to different configurations, constraints and analytical objectives.

At this stage and in parallel to encourage their deployment on an industrial scale, it is necessary to be able to upgrade them to higher Technology Readiness Level (TRL).

In addition, the results of measurements are often considered in a standalone approach as a step in itself. This greatly limits the scope of the information collected. In situ mapping in constrained environments is often a challenge and accumulated approximations in a standalone approach would have serious consequence that threaten D\&D project's completion (waste over categorization, unexpected contamination, etc.).

The reliability of a coupled approach as supported by INSIDER necessitates an assessment of the performance of measurement methods already available and without necessarily any optimization. This task is a metrological task.

\subsection{Metrology dedicated role in the project}

The project aims at improving knowledge of the radiological state of nuclear facilities under decommissioning operations in constrained environments, i.e. with limited data availability.

The heart of the technical innovative part of the project is to improve the sampling strategy coupled to in-situ and in-lab available techniques and methods, to take into account different types of measurement data, with different but known levels of accuracy [19].

Statistical approaches allow very complete simulation and risk analysis based on in-situ and in-lab measurements. To face the problem of radiological characterization in constrained environments, where very limited amount of available data is particularly challenging, and specific statistical strategy needs to be implemented in order to limit the variability and sensibility to outliers.

In-situ analysis techniques are of paramount importance for initial cartography. In constrained environment, they must be complemented by more detailed off-site measurements. Realistic knowledge of their performance is important, without systematic optimization.

An essential consideration for the INSIDER project is that it must be possible to demonstrate that any measurements carried out to characterize the waste materials are traceable to the international measurement system. Such traceability enables nuclear sites to demonstrate that measurements are accurate, fit for purpose and scientifically rigorous, to give regulators and the general public confidence in any results.

The main characterization challenges are thus:

- choosing suitable measurement techniques, well adapted to sampling requirements or expectations;

- adapting to constrained environments of corresponding in-situ and remote laboratory analysis techniques in a cost effective perspective;

- increasing the reliability of characterization data and of interpretation models.

Currently, real improvement of some analytical or measurement methods is difficult due to the lack of suitable reference materials. INSIDER will support the process development and validation of specific common reference materials and associated fabrication process.

According to previous Section 2, metrological tools should apply on:

- validation and performance assessments of methods

- thanks to specific D\&D matrix CRM developed within the project;

- benchmarking on real worksites in $\mathrm{D} \& \mathrm{D}$, consisting of in situ measurement campaign for both a mapping and an 
intercomparison measurements at specific locations, and in-lab ILC on real samples;

- uncertainty budget evaluation;

- correlation when possible between on-site global measurements and in lab RN analysis results;

- recommendations and technical methodological guides.

As already explained CRM are essential for method validation and thus, for the realistic evaluation of measurement uncertainties and for method calibration. At the present time, the only solution was to manufacture them within the project, in compliance with the materials characterized during the benchmarking. ILC on reference materials are efficient tools to estimate the performance of the measurement methods, as soon as a sufficient number of laboratories and replicates are available.

Considering an ILC gathering $\mathrm{p}$ laboratories giving $\mathrm{n}$ independent measurements, a common linear model of the results is expressed by [20] Eurachem Guide quantifying uncertainty in analytical measurement]

$$
y_{i j}=m+b_{i}+e_{i j}
$$

$y_{i j}$ being the replicate $j$ of laboratory $i, m$ the reference value or more generally a mean of all the $n \times p$ replicates results if there is no method bias, $b_{i}$ the bias due to laboratory $i$, and $e_{i j}$ the random error of laboratory $j$ under repeatability conditions.

Based on [21,22] an analysis of variance (ANOVA) can be conducted on all $y_{i j}$ measurement results to evaluate the impact of the laboratory factor $y_{i}$, assuming a Gaussian distribution of the variables. There are $p$ groups, i.e. laboratories, each of them containing $n$ replicates. The objective of an ANOVA is to determine the between (inter laboratories) and within (intra laboratory) standard deviations.

The sum of the squares of the deviations from the general mean $(m)$ is decomposed into 2 terms: one corresponding to the factor under study, the other corresponding to a residual variance. With the same numbering the model becomes:

$$
\begin{gathered}
\sum_{i=1}^{p} \sum_{j=1}^{n}\left(y_{i j}-m\right)^{2}=\sum_{i=1}^{p} \sum_{j=1}^{n}\left(\bar{y}_{i}-m\right)^{2}+\sum_{i=1}^{p} \sum_{j=1}^{n}\left(y_{i j}-\bar{y}_{i}\right)^{2} \\
\mathrm{SC}_{\text {total }}=\mathrm{SC}_{\text {Factor }}+\mathrm{SC}_{\text {residual }} .
\end{gathered}
$$

By dividing by their respective numbers of degrees of freedom $(n p-1)$ for the first term, $(p-1)$ for the second term and $p(n-1)$ for the third term), these terms correspond respectively to the total variance, the interlaboratory variance and the intralaboratory variance (residual) $s_{\text {res }}^{2}$ either:

$$
s_{R}^{2}=s_{L}^{2}+s_{\text {res }}^{2}
$$

where, $s_{R}^{2}$ is the variance of precision in reproducibility conditions, $s_{L}^{2}$ is the variance due to the laboratory factor and $s_{\text {res }}^{2}$ is the residual variance or the variance of precision in repeatability conditions.

$$
\text { With } s_{\text {res }}^{2}=\frac{\mathrm{SC}_{\text {residual }}}{p(n-1)}, s_{F}^{2}=\frac{\mathrm{SC}_{\text {factor }}}{p-1} \text { and } s_{L}^{2}=\frac{s_{F}^{2}-s_{\text {res }}^{2}}{n} \text {. }
$$

The general form of a variance analysis is based on the Fisher test and therefore on the normality of the distributions and the independence of the samples $T$.

To test the normality of the distribution, it is assumed, under the null hypothesis, that the samples come from the same population and follow a normal distribution. It is ensured by the factor $F=\frac{s_{F}^{2}}{s_{\text {res }}^{2}}$ following or not a Fisher law. The standard uncertainty is estimated by this ANOVA applied to $y_{i j}$ ILC results. This uncertainty $u(x)$ is the estimated standard uncertainty associated to measurement result obtained by the measurement method

$$
u(x)=\sqrt{s_{L}^{2}+s_{\mathrm{res}}^{2}}
$$

This approach can be extended to an analysis of two factors or multifactorial for more complex configuration, taking into account several crossed factors such as laboratory, level of activity, radionuclide.

\subsection{Validation of the original approach}

To illustrate and qualify the project-integrated approaches, the methodologies will be applied to the use cases in the form of three different benchmarking exercises on real worksites shown in Figure 2 and offered by the partners (JRC, SCKCEN and CEA).

Analytical microsystems and/or other innovative measurement devices will take part in the different ILC on CRM and on real samples (benchmarking) exercises, with increasing representability to D\&D operations [23]. They will likely benefit of an increase in TRL level for future R\&D and industrial applications. As their results are few, they are treated within the framework of PT, for an assessment and comparison of their accuracy.

The benchmark will help the project to meet some of the INSIDER project main objectives [24], in particular:

- definition of an improved sampling strategy for waste production optimization by demonstrating the feasibility in realistic cases;

- validation of rapid/cost effective analytical methods (in lab and in-situ) in realistic conditions;

- performance assessment of available measurement techniques (methods \& tools) to establish a science base for decision-making.

Among the materials most commonly found on D\&D sites, the project has focused on the treatment of activated concrete (nuclear reactor) and contaminated effluents (nuclear facility) (Tab. 1). Two activity levels were considered.

Collecting and homogenizing real samples become then paramount to improve the determination of the method performances, and identify efficiently correlations.

An ANOVA is first used to assess the performance of the analysis or measurement methods. It will confirm the ability of methods used and will assess different uncertainty components.

Two separate cases are to be consider in this part of the project:

- the ANOVA approach on the in-lab ILC, both CRM and real samples; 
Table 1. CRM characteristics from [25].

\begin{tabular}{llll}
\hline Matrix & $\mathrm{RN}$ & Interferents & $\begin{array}{l}\text { Homogeneity/ } \\
\text { granulometry }\end{array}$ \\
\hline Heavy concrete & ${ }^{133} \mathrm{Ba},{ }^{152} \mathrm{Eu},{ }^{154} \mathrm{Eu},{ }^{60}{ }^{\circ} \mathrm{Co}$ & Stable elements & $100 \mu \mathrm{m}$ \\
Aqueous solution & ${ }^{63} \mathrm{Ni},{ }^{90} \mathrm{Sr},{ }^{55} \mathrm{Fe},{ }^{238} \mathrm{Pu}$, & $\mathrm{Al}, \mathrm{B}, \mathrm{Ca}, \mathrm{Co}, \mathrm{Cr}, \mathrm{Cu}, \mathrm{Fe}, \ldots$ & $1 \mathrm{~mL}$ \\
\hline
\end{tabular}

- the ANOVA approach on the ILC on in situ measurements (real samples).

With in-lab analysis results on CRM, it gives access to the performance of the methods.

Similar methods will be applied then on real samples through ILC "benchmarking". Based on the previous performance, corrections could by applied, leading to an improved measurement result with its uncertainty for the worksite considered.

Regarding the objectives of the global integrated approach, it is of paramount importance to be able to assess the on-site measurement methods.

To overcome the difficulty of the lack of a reference value, the ILC is applied to specific pre-selected areas by each of the team participating in the on-site campaign. Measurement conditions, replicates, control of detector positioning, etc, is specified and imposed allowing an ANOVA processing to determine the global uncertainty of the methods, and in the future the contribution of the different factor [26].

In the end, depending on the range, the uncertainties thus consolidated may be transferred to all the results acquired for inclusion in the statistical data processing associated to the sampling design.

A better understanding of uncertainties also facilitates the identification of correlations between some global onsite measurement (dose rate, gamma activity,...) and main $\mathrm{RN}$ contents, including those identified as DTM.

This constitutes a second real source of mapping and modelling improvements, with a limited number of well specified analysis.

\section{Conclusion and perspectives}

The heart of the technical innovative part of the project is to improve the sampling strategy taking into account different types of measurement data, with different but known levels of accuracy. Coupling remote laboratory analysis and in situ measurement for statistical modelling is a challenge necessitating multidisciplinary developments.

The integrated concept of site radiological modeling and coupling of different characterization types will contribute to Statistical modeling to optimize sampling identified as one of the highest priority by the nuclear innovation expert group of OECD-NEA. An optimal use of existing techniques through improvement, adaptation and their validation thanks to development of specific matrix CRM is possible but facilitated by ILC within analytical networks to combine efforts and really compare the obtained results.
The experimental benchmarking supplemented by different ILC's within the project structures the validation and refinement of the INSIDER methodology. Final assessment of the outcome will strengthen the recommendations and guidance, and promote and share European expertise through guides and pre-normative texts.

The project INSIDER has received funding from the Euratom research and training program 2014-2018, under grant agreement No 755554 .

\section{Author contribution statement}

Danièle Roudil is a research Engineer at CEA with specialization in instrumentation and measurement system developments and implementation in nuclear environment. She is president of the Commission for the establishment of analytical methods at CEA Nuclear division (CETAMA) and coordinator of the INSIDER European Research and Innovation Euratom project. Marielle Crozet is a researcher at CEA with expertise in analytical chemistry, radionuclide separation and analysis, and chemical metrology. She is technical secretary at CETAMA and responsible for actinide reference solution production in the CETAMA metrology laboratory (LAMMAN). She is WP6 leader in the INSIDER European project. Sébastien PICART is a researcher at CEA with expertise in actinide chemistry and electrochemistry. $\mathrm{He}$ is technical secretary at CETAMA involved in analytical development and validation for metrology controls. Paolo Peerani is nuclear engineer at JRC. He is Head of nuclear decommissioning unit and license holder of the nuclear installations at the Ispra site, in charge of implementing the operational decommissioning and waste management programme at JRC. He is WP2 leader of the INSIDER European Project. Sven Boden is a research engineer and project leader within the Dismantling, Decontamination and Waste Expert group of the SCK•CEN. He is specialized in radiological characterization aspects related to all types of $\mathrm{D} \& \mathrm{D}$ projects, and is WP3 leader of the EU INSIDER project. $\mathrm{He}$ is the SCK•CEN representative within the NEA/ OECD Technical Advisory Group in decommissioning and the IAEA LABONET. Ben Russel is a senior radiochemist at NPL, specialising in radiochemical separation and mass spectrometric measurement of radionuclides in relation to nuclear decommissioning, naturally occurring radioactivity, forensics and nuclear medicine. He is co-coordinator of NPL's Proficiency Test Exercise programme, and is WP4 leader of the European INSIDER project. Margarita Herranz is Professor of 
Nuclear Engineering at the UPV/EHU. She is the technical director and quality manager of the Radioactivity measurements laboratory of the University (LMBA). She works mainly in the fields of radiation protection, environmental impact and metrology. She is the leader of the WP5 of the European project INSIDER. Laura Aldave de la Heras is deputy Head of the Waste management Unit and senior expert on nuclear waste management and decommissioning at JRC Directorate G Nuclear Safety and Security. She is WP7 leader of the European INSIDER project.

\section{References}

1. OCDE NEA, R\&D and innovation needs for decommissioning nuclear facilities, 2014

2. S. Boden et al., Concrete waste reduction during PWR decommissioning, in CETAMA seminar Sampling and characterization III, 2015

3. OECD-NEA, Radiological characterisation from a waste and materials end-state perspective: Practice and experience (OECD Publications, Paris, 2018)

4. D. Roudil et al., The challenges of chemical metrology in the fuel cycle, the role of CETAMA, in Global 2015 proceedings (5352)

5. C. Rivier et al., in Spectr'Atom May 2014, Pau, 2014

6. M. Crozet et al., EQRAIN: uranium and plutonium interlaboratory exercises from 1997 to 2016 - comparison to ITVs-2010, J. Radio. Nucl. Chem. 319, 1013 (2019)

7. NF ISO guide 35:2017 - Reference materials - Guidance for characterization and assessment of homogeneity and stability

8. NF ISO 17034:2016 standard: General requirements for the competence of reference material producers

9. C. Rivier et al., Use of an Excess Variance Approach for the Certification of Reference Materials by Interlaboratory comparison, J. Quality Compar. Reliab. Chem. Meas. 19, 269 (2014)
10. ISO 18557:2017 standard: characterization principles of soils, buildings and infrastructures contaminated by radionuclides for remediation purposes

11. CETAMA collective report, Evaluation of the initial and final radiological state of a nuclear facility under decommissioning CEA-R-6455 ISSN 0429-3460 (2017)

12. Y. Desnoyers et al., Data Analysis and Sampling Optimization for Radiological Characterization: Geostatistical and Statistical Complementarity, in DDER conference (2011)

13. Mesure du Rayonnement alpha, Tec\&Doc collection Lavoisier editor. ISBN: 978-2-7430-1414-8 (2012)

14. Spectrométrie gamma appliquée aux échnatillons de l'environnement, Tec\&Doc collection Lavoisier editor. ISBN: 2-7430-0580-7 (2002)

15. CETAMA collective report DROP passive neutron CEA-R6322 ISSN: 0429-3460 (2013)

16. M. Crozet et al., Sum of measurement values, Techniques de l'ingénieur, 263 (2015)

17. INSIDER project internet site: http://insider-h2020.eu/

18. S. Boden et al., R\&D within the BR3 PWR decommissioning project, in DEM 2018 international conference, Avignon (France)

19. D. Roudil et al., The European R\&D project INSIDER, in DEM 2018 international conference, Avignon (France)

20. EURACHEM/CITAC Guide CG (2012) Quantifying Uncertainty in Analytical Measurement, EURACHEM/CITAC Guide CG 4, www.eurachem.org

21. NF ISO $13528: 2015$, Méthodes statistiques utilisées dans les essais d'aptitude par comparaison interlaboratoires

22. S. Demeyer, INSIDER Mid term workshop, Bilbao, May 2019

23. S. Rasou et al., Sustainable Solvent Extraction Process for Fe Analysis in Radioactive Samples Based on Microfluidic Tools, Solvent Extraction and Ion Exchange Journal, in press

24. D. Roudil et al., The European R\&D project INSIDER: acting on the upstream stageRadWorkshop 2018, Roskilde (Danemark)

25. B. Russel, INSIDER MID term workshop, Bilbao, May 2019

26. M. Crozet, INSIDER MID term workshop, Bilbao, May 2019

Cite this article as: Danièle Roudil, Marielle Crozet, Sébastien Picart, Ben Russell, Margarita Herranz, Sven Boden, Paolo Peerani, Laura Aldave De Las Heras, Metrology applications to D\&D issues: issues at stake for insider European project, EPJ Nuclear Sci. Technol. 6, 17 (2020) 